\title{
Some Tauberian conditions on logarithmic density
}

\author{
Adem Kılıçman ${ }^{1 *} \mathbb{D}$, Stuti Borgohain² and Mehmet Küçükaslan³
}

\section{"Correspondence:}

akilic@upm.edu.my

'Department of Mathematics and Institute for Mathematical Research, University of Putra Malaysia, Serdang, Malaysia

Full list of author information is available at the end of the article

\begin{abstract}
This article is based on the study on the $\lambda$-statistical convergence with respect to the logarithmic density and de la Vallee Poussin mean and generalizes some results of logarithmic $\lambda$-statistical convergence and logarithmic $(V, \lambda)$-summability theorems. Hardy's and Landau's Tauberian theorems to the statistical convergence, which was introduced by Fast long back in 1951, have been extended by J.A. Fridy and M.K. Khan (Proc. Am. Math. Soc. 128:2347-2355, 2000) in recent years. In this article we try to generalize some Tauberian conditions on logarithmic statistical convergence and logarithmic $(V, \lambda)$-statistical convergence, and we find some new results on it.
\end{abstract}

Keywords: Statistical convergence; $\lambda$-convergence; de la Vallee Poussin mean; Logarithmic density

\section{Introduction and preliminary concepts}

In 1951, Fast [2] and Steinhaus [3] independently introduced the concept of statistical convergence for sequences of real numbers, and since then this concept has been generalized and investigated in different ways by different authors. Likewise summability theory and convergence of sequences have also been studied actively in the area of pure mathematics for the last several decades. Extensive works on the topic are applicable in topology, functional analysis, Fourier analysis, measure theory, applied mathematics, mathematical modeling, computer science, analytic number theory, etc. One may refer to [4-9], etc.

Let $A \subseteq \mathbb{N}$ and $A_{n}=\{\psi \leq n: \psi \in A\}$. We say that $A$ has natural density, i.e., $\delta(A)=$ $\lim _{n} \frac{1}{n}\left|A_{n}\right|$, if the limit exists, where $\left|A_{n}\right|$ denotes the cardinality of $A_{n}$.

By the concept of statistical convergence, we mean a sequence $\tilde{x}=\left(x_{\psi}\right)$ of real numbers which statistically converges to $\ell$ if for every $\varepsilon>0$ the set $A_{\varepsilon}=\left\{\psi \in \mathbb{N}:\left|x_{\psi}-\ell\right| \geq \varepsilon\right\}$ has natural density zero, i.e., for each $\varepsilon>0$,

$$
\lim _{n} \frac{1}{n}\left|\left\{\psi \leq n:\left|x_{\psi}-\ell\right| \geq \varepsilon\right\}\right|=0
$$

Let $\lambda=\left(\lambda_{n}\right)$ be a non-decreasing sequence of positive numbers tending to $\infty$ such that

$$
\lambda_{n+1} \leq \lambda_{n}+1
$$

and $\lambda_{1}=0$.

(c) The Author(s) 2019. This article is distributed under the terms of the Creative Commons Attribution 4.0 International License (http://creativecommons.org/licenses/by/4.0/), which permits unrestricted use, distribution, and reproduction in any medium, provided you give appropriate credit to the original author(s) and the source, provide a link to the Creative Commons license, and indicate if changes were made. 
The generalized de la Vallee Poussin mean of a sequence $\tilde{x}=\left(x_{\psi}\right)$ is defined by $T_{n}(x)=$ $\frac{1}{\lambda_{n}} \sum_{\psi \in I_{n}} x_{\psi}$, where $I_{n}=\left[n-\lambda_{n}+1, n\right]$.

Now, a sequence $\tilde{x}=\left(x_{\psi}\right)$ is said to be $(V, \lambda)$-summable to $\ell$ if $T_{n}(x)$ converges to $\ell$, i.e.,

$$
\lim _{n} \frac{1}{\lambda_{n}} \sum_{\psi \in I_{n}}\left|x_{\psi}-\ell\right|=0
$$

Also a sequence $\tilde{x}=\left(x_{\psi}\right)$ is said to be statistically $\lambda$-convergent to $\ell$ if, for every $\varepsilon>0$,

$$
\lim _{n} \frac{1}{n}\left|\left\{\psi \leq n:\left|T_{\psi}(x)-\ell\right| \geq \varepsilon\right\}\right|=0
$$

By logarithmic density, we mean $\delta_{\log _{n}}(E)=\frac{1}{\log _{n}} \sum_{\psi=1}^{n} \frac{\chi_{E}(\psi)}{\psi}$ for $E \in \mathbb{N}$, where $\log _{n}=$ $\sum_{\psi=1}^{n} \frac{1}{\psi} \approx \log n, n \in \mathbb{N}[8]$.

A sequence $\tilde{x}=\left(x_{\psi}\right)$ is logarithmic statistically convergent to $\ell$ if

$$
\lim _{n} \frac{1}{\log _{n}}\left|\left\{\psi \leq n: \frac{1}{\psi}\left|x_{\psi}-\ell\right| \geq \varepsilon\right\}\right|=0 .
$$

A sequence $\tilde{x}=\left(x_{\psi}\right)$ is logarithmic $(V, \lambda)$-statistically convergent to $\ell$ if

$$
\lim _{n} \frac{1}{\log _{\lambda_{n}}}\left|\left\{\psi \in I_{n}: \frac{1}{\psi}\left|T_{\psi}(x)-\ell\right| \geq \varepsilon\right\}\right|=0,
$$

where $\log _{\lambda_{n}}=\sum_{\psi=1}^{\lambda_{n}} \frac{1}{\psi} \approx \log \lambda_{n}(n=1,2,3, \ldots)$.

Let $\mu_{n}=\frac{1}{\log _{\lambda_{n}}} \sum_{\psi \in I_{n}} \frac{T_{\psi}(x)}{\psi}$, where $\log _{\lambda_{n}}=\sum_{\psi=1}^{\lambda_{n}} \frac{1}{\psi} \approx \log \lambda_{n}(n=1,2,3, \ldots)$. A sequence $\tilde{x}=\left(x_{\psi}\right)$ is logarithmic $(V, \lambda)$-summable to $\ell$ if $\left(\mu_{n}\right)$ is convergent to $\ell$, i.e., $\lim _{n} \frac{1}{\log _{\lambda_{n}}} \sum_{\psi \in I_{n}} \frac{\left|T_{\psi}(x)-\ell\right|}{\psi}=0$

A sequence $\tilde{x}=\left(x_{\psi}\right)$ is logarithmic $(V, \lambda)$-statistically summable to $\ell$ if $\left(\mu_{n}\right)$ is $\lambda$ statistically convergent, i.e.,

$$
\lim _{n} \frac{1}{\lambda_{n}}\left|\left\{\psi \in I_{n}:\left|\mu_{\psi}-\ell\right| \geq \varepsilon\right\}\right|=0 .
$$

We define it as $s t_{\log _{\lambda_{n}}}-\lim _{n} T_{n}=\ell$.

Moricz [10] studied the concept of Tauberian conditions for statistical convergence followed from statistical summability $(C, 1)$. Braha [11] extended these results using Tauberian conditions for $\lambda$-statistical convergence, which was followed from statistical summability $(V, \lambda)$. Braha [12] also explained the Tauberian theorems for the generalized Norlund-Euler summability method. One may refer to [13-15].

In this paper, we study the Tauberian theorems for logarithmic $(V, \lambda)$-statistical convergence which is followed from de la Vallee Poussin mean. We also try to establish some results involving the logarithmic density.

\section{Main results}

Theorem 2.1 Let $\lambda$ be a real-valued sequence defined in (1). Then,

1. If $\tilde{x}=\left(x_{\psi}\right)$ is logarithmic $(V, \lambda)$-statistically summable to $\ell$, then it is logarithmic $(V, \lambda)$-statistically convergent to $\ell$, provided $\liminf _{n} \frac{1}{\lambda_{n}}>0$. 
2. If $\tilde{x}=\left(x_{\psi}\right)$ is bounded, then logarithmic $(V, \lambda)$-statistical convergence implies logarithmic $(V, \lambda)$-statistical summability.

3. $\Omega\left(\log _{n}, \lambda\right) \cap \ell_{\infty}=\Pi\left(\log _{n}, \lambda\right)$, where $\Omega\left(\log _{n}, \lambda\right)$ is the collection of all logarithmic $(V, \lambda)$-statistical convergence sequences, $\ell_{\infty}$ is the collection of all bounded sequences, and $\Pi\left(\log _{n}, \lambda\right)$ is the collection of all logarithmic $(V, \lambda)$-summable sequences.

Proof (1) Let $\tau_{n}=\left\{\psi \in I_{n}: \frac{1}{\log _{\lambda_{n}}} \sum_{\psi \in I_{n}} \frac{1}{\psi}\left|T_{\psi}(x)-\ell\right| \geq \varepsilon\right\}$.

Since $\tilde{x}=\left(x_{\psi}\right)$ is logarithmic $(V, \lambda)$-statistically summable to $\ell$, then $\tau_{n}$ is $\lambda$-statistically convergent to $\ell$, i.e.,

$$
\lim _{n} \frac{1}{\lambda_{n}}\left|\left\{\psi \in I_{n}: \frac{1}{\log _{\lambda_{n}}} \sum_{\psi \in I_{n}} \frac{1}{\psi}\left|T_{\psi}(x)-\ell\right| \geq \varepsilon\right\}\right|=0 .
$$

Also we can write

$$
\begin{aligned}
\frac{1}{\log _{\lambda_{n}}} \sum_{\psi \in I_{n}} \frac{1}{\psi}\left|T_{\psi}(x)-\ell\right| & \geq \frac{1}{\log _{\lambda_{n}}} \sum_{\psi \in I_{n},\left|\frac{T_{\psi}(x)}{\psi}-\ell\right| \geq \varepsilon} \frac{1}{\psi}\left|T_{\psi}(x)-\ell\right| \\
& \geq \frac{1}{\log _{\lambda_{n}}}\left(\left|\left\{\psi \in I_{n}: \frac{1}{\psi}\left|T_{\psi}(x)-\ell\right| \geq \varepsilon\right\}\right| \varepsilon\right),
\end{aligned}
$$

which implies that

$$
\frac{1}{\lambda_{n}}\left\{\psi \in I_{n}: \frac{1}{\log _{\lambda_{n}}} \sum_{\psi \in I_{n}} \frac{1}{\psi}\left|T_{\psi}(x)-\ell\right| \geq \varepsilon\right\} \geq \frac{1}{\lambda_{n}} \frac{\left(\left|\left\{\psi \in I_{n}: \frac{1}{\psi}\left|T_{\psi}(x)-\ell\right| \geq \varepsilon\right\}\right| \varepsilon\right)}{\log _{\lambda_{n}}} .
$$

Since $\liminf _{n} \frac{1}{\lambda_{n}}>0$ and $\tilde{x}=\left(x_{\psi}\right)$ is logarithmic $(V, \lambda)$-statistically summable to $\ell$, so by taking $n \rightarrow \infty$, we get $\tilde{x}=\left(x_{\psi}\right)$ is logarithmic $(V, \lambda)$-statistically convergent to $\ell$. This completes the proof.

Proof (2) Let $\tilde{x}=\left(x_{\psi}\right)$ be bounded and logarithmic $(V, \lambda)$-statistically convergent to $\ell$. Then there exists $M>0$ such that $\left|x_{\psi}-\ell\right| \leq M$. Now, for any $\varepsilon>0$,

$$
\begin{aligned}
\frac{1}{\log _{\lambda_{n}}} \sum_{\psi \in I_{n}} \frac{1}{\psi}\left|T_{\psi}(x)-\ell\right|= & \frac{1}{\log _{\lambda_{n}}} \sum_{\psi \in I_{n}, \psi \notin B(n)} \frac{1}{\psi}\left|T_{\psi}(x)-\ell\right| \\
& +\frac{1}{\log _{\lambda_{n}}} \sum_{\psi \in I_{n}, \psi \in B(n)} \frac{1}{\psi}\left|T_{\psi}(x)-\ell\right| \\
= & K_{1}(n)+K_{2}(n),
\end{aligned}
$$

where $B(n)=\left\{\psi \in I_{n}: \frac{1}{\psi}\left|T_{\psi}(x)-\ell\right| \geq \varepsilon\right\}$

Now, if $\psi \notin B(n)$, then $K_{1}(n)<\varepsilon$. For $\psi \in B(n)$, we have

$$
K_{2}(n) \leq\left(\sup \left|T_{\psi}(x)-\ell\right|\right) \cdot\left(|B(n)| / \log _{\lambda_{n}}\right) \leq M|B(n)| / \log _{\lambda_{n}} \rightarrow 0
$$

as $n \rightarrow \infty$.

Since logarithmic density of $B(n)$ is zero, hence we can say that $\tilde{x}=\left(x_{\psi}\right)$ is logarithmic $(V, \lambda)$-statistically summable. This completes the proof. 
Proof Proof of (3) follows from the proof of (1) and (2), so it is omitted here.

\section{Tauberian theorems}

Theorem 3.1 Let $\left(\lambda_{n}\right)$ be a sequence of real numbers and $s t_{\log _{\lambda_{n}}}-\lim _{n} \inf \frac{\lambda t_{n}}{\lambda_{n}}>1$ for all $t>1$, where $t_{n}$ denotes the integral parts of $[t . n]$ for every $n \in \mathbb{N}$, and let $\left(T_{\psi}\right)$ be a sequence of real numbers such that $t_{\log _{\lambda_{n}}}-\lim _{n} T_{n}=\ell$. Then $\tilde{x}=\left(x_{\psi}\right)$ is st $t_{\log _{\lambda_{n}}}$-convergent to $\ell$ iff the following conditions hold:

$$
\inf _{t>1} \lim _{n} \sup \frac{1}{\log _{\lambda_{n}}}\left|\left\{\psi \in I_{n}: \frac{1}{\lambda_{t_{\psi}}-\lambda_{\psi}} \sum_{j=\psi+1}^{t_{\psi}} \frac{1}{\psi}\left(x_{j}-x_{\psi}\right) \leq-\varepsilon\right\}\right|=0
$$

and

$$
\inf _{0<t<1} \limsup _{n} \frac{1}{\log _{\lambda_{n}}}\left|\left\{\psi \in I_{n}: \frac{1}{\lambda_{\psi}-\lambda_{t_{\psi}}} \sum_{j=t_{\psi}+1}^{k} \frac{1}{\psi}\left(x_{\psi}-x_{j}\right) \leq-\varepsilon\right\}\right|=0 \text {. }
$$

Remark Let us suppose that

$$
s t_{\log _{\lambda_{n}}}-\lim _{n} x_{n}=\ell \quad \text { and } \quad s t_{\log _{\lambda_{n}}}-\lim _{n} T_{n}=\ell
$$

are satisfied, then for every $t>1$, the following relation is valid:

$$
s t_{\log _{\lambda_{n}}}-\lim _{n} x_{n}=\ell \text { implies that } \lim _{n} \frac{1}{\log _{\lambda_{n}}} \sum_{\psi=1}^{n} \frac{1}{\psi}\left|x_{\psi}-\ell\right|=0
$$

and

$$
s t_{\log _{\lambda_{n}}}-\lim _{n} T_{n}=\ell \quad \text { implies that } \lim _{n} \frac{1}{\log _{\lambda_{n}}} \sum_{\psi=1}^{n} \frac{1}{\psi}\left|T_{\psi}(x)-\ell\right|=0 \text {, }
$$

from which it follows that $s t_{\lambda}-\lim _{n} \frac{1}{\log _{\left(\lambda_{t_{\psi}}-\lambda_{\psi}\right)}} \sum_{\psi=n+1}^{t_{n}} \frac{1}{\psi} x_{\psi}=0$ holds for $t>1$, i.e.,

$$
\lim _{n} \frac{1}{\log _{\lambda_{n}}}\left|\left\{\psi \leq n: \frac{1}{\lambda_{t_{\psi}}-\lambda_{\psi}} \sum_{\psi=1}^{t_{n}} \frac{\left|x_{\psi}\right|}{\psi} \geq \varepsilon\right\}\right|=0,
$$

and for $0<t<1$, we have $s t_{\lambda}-\lim _{n} \frac{1}{\lambda_{\psi}-\lambda_{t_{\psi}}} \sum_{\psi=t_{n}+1}^{n} \frac{x_{\psi}}{\psi}=0$, i.e.,

$$
\lim _{n} \frac{1}{\log _{\lambda_{n}}}\left|\left\{\psi \leq n: \frac{1}{\lambda_{\psi}-\lambda_{t_{\psi}}} \sum_{\psi=t_{n}+1}^{n} \frac{\left|x_{\psi}\right|}{\psi} \geq \varepsilon\right\}\right|=0
$$

holds.

Lemma 3.1 For the sequence of real numbers $\lambda=\left(\lambda_{n}\right)$, (2) is equivalent to $s t_{\lambda_{n}}-$ $\lim _{n} \inf \frac{\lambda_{n}}{\lambda_{t_{n}}}>1$ for all $0<t<1[12]$. 
Lemma 3.2 If $s t_{\log _{\lambda_{n}}}-\lim _{n} x_{n}=\ell$ and $s t_{\log _{\lambda_{n}}}-\lim _{n} T_{n}=\ell$ are satisfied, and let $\tilde{x}=\left(x_{\psi}\right)$ be a sequence of complex numbers which is logarithmic $(V, \lambda)$-statistically convergent to $\ell$, then for any $t>1$,

$$
s t_{\log _{\lambda_{n}}}-\lim _{n} T_{t_{n}}=\ell, \quad \text { i.e., } \quad \frac{1}{\log _{\lambda_{n}}}\left|\left\{\psi \leq n: \sum_{\psi=1}^{n} \frac{1}{\psi}\left|T_{t_{\psi}}-\ell\right| \geq \varepsilon\right\}\right|=0 .
$$

Proof Case I: Let us consider that $t>1$, then from construction of the sequence $\lambda=\left(\lambda_{n}\right)$ we get

$$
\lim _{n}\left(n-\lambda_{n}\right)=\lim _{n}\left(t_{n}-\lambda_{t_{n}}\right)
$$

and for every $\varepsilon>0$, we have

$$
\begin{aligned}
& \left\{\psi \in I_{t_{n}}: \frac{1}{\psi}\left|T_{t_{\psi}}-\ell\right| \geq \varepsilon\right\} \\
& \quad \subset\left\{\psi \in I_{n}: \frac{1}{\psi}\left|T_{\psi}-L\right| \geq \varepsilon\right\} \cup\left\{\psi \in I_{n}: \frac{1}{\log _{\lambda_{\psi}}} \sum_{j=\psi-\lambda_{\psi}+1}^{\psi} \frac{x_{j}}{j} \neq \frac{1}{\log _{\lambda_{t_{\psi}}}} \sum_{j=t_{\psi}-\lambda_{t_{\psi}}+1}^{t_{\psi}} \frac{x_{j}}{j}\right\} .
\end{aligned}
$$

Following Eq. (3), we can say that $s t_{\log _{\lambda}}-\lim T_{t_{n}}=\ell$.

Case II: Now suppose that $0<t<1$. For the definition of $t_{n}=[t . n]$, for any natural number $n$, we can conclude that $\left(T_{t_{n}}\right)$ does not appear more than $\left[1+t^{-1}\right]$ times in the sequence $\left(T_{n}\right)$. In fact, if there exist integers $\psi, m$ such that

$$
n \leq t . \psi<t(\psi+1)<\cdots<t(\psi+m-1)<n+1 \leq t(\psi+m),
$$

then

$$
n+t(m-1) \leq t(\psi+m-1)<n+1 \quad \Rightarrow \quad m<1+\frac{2}{t} .
$$

So, we have the following inequality:

$$
\begin{aligned}
\frac{1}{\log _{\lambda_{t_{n}}}}\left|\left\{\psi \in I_{t_{n}}: \frac{1}{\psi}\left|T_{t_{\psi}}-\ell\right| \geq \varepsilon\right\}\right| & \leq\left(1+\frac{1}{t}\right) \frac{1}{\log _{\lambda_{t_{n}}}}\left|\left\{\psi \in I_{n}: \frac{1}{\psi}\left|T_{\psi}-\ell\right| \geq \varepsilon\right\}\right| \\
& \leq 2(1+t) \frac{1}{\log _{\lambda_{n}}}\left|\left\{\psi \in I_{n}: \frac{1}{\psi}\left|T_{\psi}-\ell\right| \geq \varepsilon\right\}\right|,
\end{aligned}
$$

which gives that $s t_{\log _{\lambda_{n}}}-\lim _{n} T_{t_{n}}=\ell$.

Lemma 3.3 If st $\log _{\lambda_{n}}-\lim _{n} x_{n}=\ell$ and $s t_{\log _{\lambda_{n}}}-\lim _{n} T_{n}=\ell$ are satisfied and $\tilde{x}=\left(x_{\psi}\right)$ is logarithmic $(V, \lambda)$-statistically convergent to $\ell$, then we have

(i) $\lim _{n} \frac{1}{\log _{\lambda_{n}}}\left|\left\{\psi \leq n: \frac{1}{\lambda_{t_{n}}-\lambda_{n}} \sum_{\psi=n+1}^{t_{n}} \frac{\left|x_{\psi}-\ell\right|}{\psi} \geq \varepsilon\right\}\right|=0 \quad$ for every $t>1$ 
and

(ii) $\lim _{n} \frac{1}{\log _{\lambda_{n}}}\left|\left\{\psi \leq n: \frac{1}{\lambda_{n}-\lambda_{t_{n}}} \sum_{\psi=t_{n}+1}^{n} \frac{1}{\psi}\left|x_{\psi}-\ell\right| \geq \varepsilon\right\}\right|=0 \quad$ for any $0<t<1$.

Proof (i) Let us suppose that $t>1$. We get

$$
\begin{aligned}
\frac{1}{\lambda_{t_{n}}-\lambda_{n}} \sum_{j=n+1}^{t_{n}} \frac{x_{j}}{j}= & T_{n}+\frac{\lambda_{t_{n}}}{\lambda_{t_{n}}-\lambda_{n}}\left(T_{t_{n}}-T_{n}\right) \\
& +\frac{1}{\lambda_{t_{n}}-\lambda_{n}} \sum_{j=n-\lambda_{n}+1}^{t_{n}} \frac{x_{j}}{j}-\frac{1}{\lambda_{t_{n}}-\lambda_{n}} \sum_{j=t_{n}-\lambda_{t_{n}}+1}^{t_{n}} \frac{x_{j}}{j} \\
\Rightarrow \quad \frac{1}{\lambda_{t_{n}}-\lambda_{n}} \sum_{j=n+1}^{t_{n}} \frac{x_{j}}{j}= & T_{n}+\frac{\lambda_{t_{n}}}{\lambda_{t_{n}}-\lambda_{n}}\left(T_{t_{n}}-T_{n}\right) \\
& \quad+\frac{1}{\lambda_{t_{n}}-\lambda_{n}}\left(\sum_{j=n-\lambda_{n}+1}^{t_{n}} \frac{x_{j}}{j}-\sum_{j=t_{n}-\lambda_{t_{n}}+1}^{t_{n}} \frac{x_{j}}{j}\right) .
\end{aligned}
$$

From the definition of the sequence $\left(\lambda_{n}\right)$ and logarithmic density, we obtain

$$
s t_{\log _{\lambda_{n}}}-\limsup _{n} \sum_{j=n-\lambda_{n}+1}^{t_{n}} x_{j}=s t_{\log _{\lambda_{n}}}-\limsup _{n} \sum_{j=t_{n}-\lambda_{t_{n}}+1}^{t_{n}} x_{j} .
$$

Let us suppose that $s t_{\log _{\lambda_{n}}}-\lim _{n} \sup \sum_{j=n-\lambda_{n}+1}^{t_{n}} x_{j}=L$, and for every $\varepsilon>0$, we get

$$
\begin{gathered}
\frac{\lim _{n} \frac{1}{\log _{\lambda_{n}}}\left|\left\{\psi \in I_{t_{n}}:\left|\sum_{j=t_{\psi}-\lambda_{t_{\psi}}+1}^{t_{\psi}} \frac{\left|x_{j}-\ell\right|}{j}\right| \geq \varepsilon\right\}\right|}{\lambda_{t_{n}}} \\
\leq \frac{\lim _{n} \frac{1}{\log _{\lambda_{n}}}\left|\left\{\psi \in I_{n}:\left|\sum_{j=\psi-\lambda_{\psi}+1}^{t_{\psi}} \frac{\left|x_{j}-\ell\right|}{j}\right| \geq \varepsilon\right\}\right|}{\lambda_{n}} \\
+\frac{\lim _{n} \frac{1}{\log _{\lambda_{n}}}\left|\left\{\psi \in I_{n}: \sum_{j=t_{\psi}-\lambda_{t_{\psi}}+1}^{t_{\psi}} \frac{x_{j}}{j} \not \sum_{j=\psi-\lambda_{\psi}+1}^{t_{\psi}} \frac{x_{j}}{j}\right\}\right|}{\lambda_{n}},
\end{gathered}
$$

from which it follows that $s t_{\log _{\lambda_{n}}}-\lim _{n} \sup \sum_{j=t_{n}-\lambda_{t_{n}+1}}^{t_{n}} x_{j}=L$.

Also, since $s t_{\lambda}-\lim _{n} \sup \frac{\lambda_{t_{n}}}{\lambda_{t_{n}}-\lambda_{n}}<\infty$ and $s t_{\lambda}-\lim _{n} \sup \frac{1}{\lambda_{t_{n}}-\lambda_{n}}<\infty$, then we get

$$
s t_{\log _{\lambda_{n}}}-\lim _{n} \frac{1}{\lambda_{t_{n}}-\lambda_{n}} \sum_{j=n+1}^{t_{n}} x_{j}=\ell .
$$

(ii) If $0<t<1$, we have

$$
\frac{1}{\lambda_{n}-\lambda_{t_{n}}} \sum_{j=t_{n}+1}^{n} \frac{x_{j}}{j}=T_{n}+\frac{\lambda_{t_{n}}}{\lambda_{n}-\lambda_{t_{n}}}\left(T_{n}-T_{t_{n}}\right)+\frac{1}{\lambda_{n}-\lambda_{t_{n}}} \sum_{j=n-\lambda_{n}+1}^{n} \frac{x_{j}}{j}-\frac{1}{\lambda_{t_{n}}-\lambda_{n}} \sum_{j=t_{n}-\lambda_{t_{n}}+1}^{n} \frac{x_{j}}{j} .
$$

This completes the proof. 
Following the above procedure, we can get the proof of Theorem 3.1.

Proof of Theorem 3.1 Let us suppose that $s t_{\log _{\lambda}}-\lim _{\psi} x_{\psi}=L$ and $s t_{\log _{\lambda}}-\lim _{\psi} T_{\psi}=\ell$. For every $t>1$, we get (by Lemma 3.2)

$$
\inf _{t>1} \limsup _{n} \frac{1}{\log _{\lambda_{n}}}\left|\left\{\psi \in I_{n}: \frac{1}{\lambda_{t_{\psi}}-\lambda_{\psi}} \sum_{j=\psi+1}^{t_{\psi}} \frac{1}{\psi}\left(x_{j}-x_{\psi}\right) \leq-\varepsilon\right\}\right|=0 .
$$

Similarly, if $0<t<1$, we obtain (by Lemma 3.2)

$$
\inf _{0<t<1} \lim _{n} \sup \frac{1}{\log _{\lambda_{n}}}\left|\left\{\psi \in I_{n}: \frac{1}{\lambda_{\psi}-\lambda_{t_{\psi}}} \sum_{j=t_{\psi}+1}^{\psi} \frac{1}{\psi}\left(x_{\psi}-x_{j}\right) \leq-\varepsilon\right\}\right|=0
$$

Now assume that $s t_{\log _{\lambda}}-\lim _{n} T_{n}=\ell$ and

$$
\begin{aligned}
& s t_{\log _{\lambda_{n}}}-\liminf _{n} \frac{\lambda_{t_{n}}}{\lambda_{n}}>1 \quad \text { for all } t>1, \\
& \inf _{t>1} \lim _{n} \sup \frac{1}{\log _{\lambda_{n}}}\left|\left\{\psi \in I_{n}: \frac{1}{\lambda_{t_{\psi}}-\lambda_{\psi}} \sum_{j=\psi+1}^{t_{\psi}} \frac{1}{\psi}\left(x_{j}-x_{\psi}\right) \leq-\varepsilon\right\}\right|=0,
\end{aligned}
$$

and

$$
\inf _{0<t<1} \limsup _{n} \frac{1}{\log _{\lambda_{n}}}\left|\left\{\psi \in I_{n}: \frac{1}{\lambda_{\psi}-\lambda_{t_{\psi}}} \sum_{j=t_{\psi}+1}^{\psi} \frac{1}{\psi}\left(x_{\psi}-x_{j}\right) \leq-\varepsilon\right\}\right|=0
$$

are satisfied. We have to prove that $s t_{\log _{\lambda}}-\lim _{n} x_{n}=\ell$ or equivalently $s t_{\log _{\lambda}}-$ $\lim _{n}\left(T_{n}-x_{n}\right)=0$.

Case I: If $t>1$, let us suppose

$$
x_{n}-T_{n}=\lambda_{t_{n}} \frac{T_{t_{n}}-T_{n}}{\lambda_{t_{n}}-\lambda_{n}}-\frac{1}{\lambda_{t_{n}}-\lambda_{n}} \sum_{j=n+1}^{t_{n}} \frac{x_{j}-x_{n}}{j} .
$$

For any $\varepsilon>0$, we obtain

$$
\begin{aligned}
& \left\{\psi \in I_{n}: x_{n}-T_{n} \geq \varepsilon\right\} \\
& \quad \subset\left\{\psi \in I_{n}: \frac{\lambda_{t_{n}}}{\lambda_{t_{n}}-\lambda_{n}}\left(T_{t_{n}}-T_{n}\right) \geq \frac{\varepsilon}{2}\right\} \cup\left\{\psi \in I_{n}: \frac{1}{\lambda_{t_{n}}-\lambda_{n}} \sum_{j=n+1}^{t_{n}} \frac{x_{j}-x_{\psi}}{j} \leq \frac{-\varepsilon}{2}\right\} .
\end{aligned}
$$

From the above relation (9), it follows that, for any arbitrary $\gamma>0$, there exists $t>1$ such that

$$
\limsup _{n} \frac{1}{\log _{\lambda_{n}}}\left|\left\{\psi \in I_{n}: \frac{1}{\lambda_{t_{\psi}}-\lambda_{\psi}} \sum_{j=\psi+1}^{t_{\psi}} \frac{1}{x_{j}-x_{\psi}} j \leq-\varepsilon\right\}\right| \leq \gamma .
$$


Also following Lemma 3.2 and the relations $s t_{\lambda}-\lim _{n} \sup \frac{\lambda_{t_{n}}}{\lambda_{t_{n}}-\lambda_{n}}<\infty$ and $s t_{\lambda}-$ $\lim _{n} \sup \frac{1}{\lambda_{t_{n}}-\lambda_{n}}<\infty$, we get

$$
\limsup _{n} \frac{1}{\log _{\lambda_{n}}}\left|\left\{\psi \in I_{n}:\left|\frac{\lambda_{t_{n}}}{\lambda_{t_{n}}-\lambda_{n}} T_{t_{n}}-T_{n}\right| \geq \frac{\varepsilon}{2}\right\}\right|=0
$$

Combining these relations, we have

$$
\limsup _{n} \frac{1}{\log _{\lambda_{n}}}\left|\left\{\psi \in I_{n}: \sum_{j} \frac{x_{n}-T_{n}}{j} \geq \varepsilon\right\}\right| \leq \gamma
$$

Since $\gamma$ is arbitrary, we conclude that, for every $\varepsilon>0$,

$$
\limsup _{n} \frac{1}{\log _{\lambda_{n}}}\left|\left\{\psi \in I_{n}: \sum_{j} \frac{x_{n}-T_{n}}{j} \geq \varepsilon\right\}\right|=0
$$

Case II: If $0<t<1$, let us suppose

$$
x_{n}-T_{n}=\lambda_{t_{n}} \frac{T_{t_{n}}-T_{n}}{\lambda_{n}-\lambda_{t_{n}}}-\frac{1}{\lambda_{n}-\lambda_{t_{n}}} \sum_{j=t_{n}+1}^{n} \frac{x_{n}-x_{j}}{j} .
$$

For any $\varepsilon>0$,

$$
\begin{aligned}
& \left\{\psi \in I_{n}: x_{n}-T_{n} \leq-\varepsilon\right\} \\
& \quad \subset\left\{\psi \in I_{n}: \frac{\lambda_{t_{n}}}{\lambda_{n}-\lambda_{t_{n}}}\left(T_{n}-T_{t_{n}}\right) \leq-\frac{\varepsilon}{2}\right\} \cup\left\{\psi \in I_{n}: \frac{1}{\lambda_{n}-\lambda_{t_{n}}} \sum_{j=t_{n}+1}^{n} \frac{x_{\psi}-x_{j}}{j} \leq-\frac{\varepsilon}{2}\right\} .
\end{aligned}
$$

Proceeding in the same way as above, we get the result as follows:

$$
\lim _{n} \frac{1}{\log _{\lambda_{n}}}\left|\left\{\psi \in I_{n}: \sum_{n} \frac{x_{n}-T_{n}}{n} \geq \varepsilon\right\}\right|=0
$$

This completes the proof of the theorem.

Theorem 3.2 Let $\left(\lambda_{n}\right)$ be a sequence of complex numbers which satisfies the following condition:

$$
s t_{\log _{\lambda_{n}}}-\liminf _{n} \frac{\lambda_{t_{n}}}{\lambda_{n}}>1 \quad \text { for all } t>1
$$

and also consider that $s t_{\log _{\lambda}}-\lim T_{\psi}=\ell$. Then $\left(x_{\psi}\right)$ is st $\log _{\lambda}$-statistically convergent to the same number $\ell$ if and only if the following two conditions hold: for every $\varepsilon>0$,

$$
\inf _{t>1} \limsup _{n} \frac{1}{\log _{\lambda_{n}}}\left|\left\{\psi \in I_{n}: \frac{1}{\lambda_{t_{\psi}}-\lambda_{\psi}} \sum_{j=\psi+1}^{t_{\psi}} \frac{x_{j}-x_{\psi}}{j} \geq \varepsilon\right\}\right|=0
$$


and

$$
\inf _{0<t<1} \lim _{n} \sup \frac{1}{l_{\lambda_{n}}}\left|\left\{\psi \in I_{n}: \frac{1}{\lambda_{\psi}-\lambda_{t_{\psi}}} \sum_{j=t_{\psi}+1}^{\psi} \frac{x_{\psi}-x_{j}}{j} \geq \varepsilon\right\}\right|=0 \text {. }
$$

Proof Proofs can be obtained by following Theorem 3.1.

\section{Conclusion}

In this paper, the Tauberian conditions under the logarithmic statistical convergence following from $(V, \lambda)$-summability are studied. The Tauberian conditions can be further applied in probabilistic normed linear spaces with $f$-density. They can also be studied in the approximation theorem point of view in more extended forms.

\section{Acknowledgements}

The authors thank the referees for their constructive comments that improved the quality of the work.

\section{Funding}

Not applicable.

\section{Availability of data and materials}

Not applicable.

\section{Competing interests}

The authors declare that they have no conflict of interests.

Authors' contributions

The authors contributed equally. All authors read and approved the final copy of manuscript.

\section{Author details}

${ }^{1}$ Department of Mathematics and Institute for Mathematical Research, University of Putra Malaysia, Serdang, Malaysia. ${ }^{2}$ Department of Mathematics, Institute of Chemical Technology, Mumbai, India. ${ }^{3}$ Department of Mathematics, Mersin University, Mersin, Turkey.

\section{Publisher's Note}

Springer Nature remains neutral with regard to jurisdictional claims in published maps and institutional affiliations.

Received: 7 August 2019 Accepted: 26 September 2019 Published online: 07 October 2019

\section{References}

1. Fridy, J.A., Khan, M.K.: Statistical extensions of some classical Tauberian theorems. Proc. Am. Math. Soc. 128, 2347-2355 (2000)

2. Fast, H.: Sur la convergence statistique. Colloq. Math. 2, 241-244 (1951)

3. Steinhaus, H.: Sur la convergence ordinaire et la convergence asymptotique. Colloq. Math. 2, 73-84 (1951)

4. Savaş, E., Borgohin, S.: On strongly almost lacunary statistical A-convergence and lacunary A-statistical convergence. Filomat 30(3), 689-697 (2016). https://doi.org/10.2298/FIL1603689S

5. Fridy, J.A.: On statistical convergence. Analysis 24, 127-145 (2004)

6. Mursaleen, M., Alotaibi, A.: Statistical summability and approximation by de la Vallee-Poussin mean. Appl. Math. Lett. 24, 320-324 (2011)

7. Mursaleen, M: $\lambda$-Statistical convergence. Math. Slovaca 50, 111-115 (2000)

8. Alghamdi, M.A., Mursaleen, M., Alotaibi, A.: Logarithmic density and logarithmic statistical convergence. Adv. Differ. Equ. 2013, 227 (2013). https://doi.org/10.1186/1687-1847-2013-227

9. Šalát, T:: On statistically convergent sequences of real numbers. Math. Slovaca 30, 139-150 (1980)

10. Moricz, F.: Tauberian conditions, under which statistical convergence follows from statistical summability $(C, 1)$. J. Math. Anal. Appl. 275, 277-287 (2002)

11. Braha, N.L.: Tauberian conditions under which $\lambda$-statistical convergence follows from statistical summability $(V, \lambda)$ Miskolc Math. Notes 16(20), 695-703 (2015)

12. Braha, N.L.: A Tauberian theorem for the generalized Norlund-Euler summability method. J. Inequal. Spec. Funct. 7(4), 137-142 (2016)

13. Belen, C.: Some Tauberian conditions obtained through weighted generator sequences. Georgian Math. J. 21(4), 407-413 (2014). https://doi.org/10.1515/gmj-2014-0040

14. Chen, C.P., Chang, C.T.: Tauberian conditions under which the original convergence of double sequences follows from the statistical convergence of their weighted means. J. Math. Anal. Appl. 332, 1242-1248 (2007)

15. Edeley, O., Mursaleen, M.: Tauberian theorems for statistically convergent double sequences. Inf. Sci. 176(7), 875-886 (2006). https://doi.org/10.1016/j.ins.2005.01.006 